\title{
Uncertainty Analysis for Wheelchair Propulsion Dynamics
}

\author{
Rory A. Cooper, Senior Member, IEEE, Michael L. Boninger, David P. VanSickle, \\ Rick N. Robertson, Member, IEEE, and Sean D. Shimada
}

\begin{abstract}
Wheelchair propulsion kinetic measurements require the use of custom pushrim force/moment measuring instruments which are not currently commercially available. With the ability to measure pushrim forces and moments has come the development of several dynamic metrics derived for analyzing key aspects of wheelchair propulsion. This paper presents several of the equations used to calculate or derive the primary variables used in the study of wheelchair propulsion biomechanics. The uncertainties for these variables were derived, and then numerically calculated for a current version of the SMART ${ }^{\text {Wheel }}$. The uncertainty results indicate that the SMART ${ }^{\text {Wheel }}$ provides data which has better than 5 to $10 \%$ uncertainty, depending upon the variable concerned, at the maximum, and during most of the propulsion phase the uncertainty is considerably smaller (i.e., approximately $1 \%$ ). The uncertainty analysis provides a more complete picture of the attainable accuracy of the SMART ${ }^{\text {Wheel }}$ and of the degree of confidence with which the data can be recorded. The derivations and results indicate where improvements in measurement of wheelchair propulsion biomechanical variables are likely to originate. The most efficient approach is to address those variables in the design of the system which make the greatest contribution to the uncertainty. Future research will focus on the point of force application and examination of nonlinear effects.
\end{abstract}

$\begin{array}{ll} & \text { NOMENCLATURE } \\ & \text { LIST OF VARIABLES } \\ p & \text { General data reduction function. } \\ U_{p} & \text { Uncertainty of } p . \\ q_{i} & \text { General independent variables which } \\ & \text { form } p . \\ \gamma_{i} & \text { Absolute sensitivty coefficient. }\end{array}$

Manuscript received September 18, 1996; revised March 26, 1997. This work was supported in part by a U.S. Department of Veterans Affairs, Rehabilitation Research and Development Service, Merit Review Grant (B686RA) and the National Center for Medical Rehabilitation Research, National Institute for Child and Human Development, National Institutes of Health (1 KO8 HD01122-01).

R. A. Cooper, M. L. Boninger, and R. N. Robertson are with the Human Engineering Research Laboratories, Departments of Rehabilitation Science and Technology, School of Health and Rehabilitation Sciences, Mechanical Engineering and Bioengineering, School of Engineering, University of Pittsburgh, Pittsburgh, PA 15206 USA. They are also with the Division of Physical Medicine and Rehabilitation, Department of Orthopaedic Surgery, University of Pittsburgh Medical Center, Veterans Affairs Medical Center, Pittsburgh, PA 15206 USA.

D. P. VanSickle and S. D. Shimada are with the Human Engineering Research Laboratories, Departments of Rehabilitation Science and Technology, School of Health and Rehabilitation Sciences, Mechanical Engineering and Bioengineering, School of Engineering, University of Pittsburgh, Veterans Affairs Medical Center, Pittsburgh, PA 15206 USA.

Publisher Item Identifier S 1063-6528(97)04554-0.
$\theta$
$r$
$F_{x}$
$F_{y}$
$F_{z}$
$v_{i}$
$k_{i}$
$M_{x}$
$M_{y}$
$M_{z}$
$\varphi$
$m_{x}$
$m_{y}$
$F_{r}$
$F_{t}$
$\beta$
$\alpha$
$F_{x_{c}}, F_{y_{c}}, F_{z_{c}}$
$F_{x_{a}}, F_{y_{a}}, F_{z_{a}}$
$F_{x_{c, a}}, F_{y_{c, a}}, F_{z_{c, a}}$
Angle of reference beam with respect to top dead center.
Radius of pushrim.
Pushrim force along the horizontal axis with the positive direction being along the line of forward progression.
Pushrim force along the vertical axis with positive being outward.
Pushrim force along the medial-lateral axis with positive being outward.
Voltages from strain gauge bridges af- ter amplification.
Calibration constants to convert volt- ages to newtons.
Pushrim moment about the $x$-axis.
Pushrim moment about the $y$-axis.
Pushrim moment about the $z$-axis.
Point of force application angle with respect to horizontal (i.e., $x$-axis).
Wrench moment applied by the hand to the pushrim about $x$-axis.
Wrench moment applied by the hand to the pushrim about $y$-axis.
Radial force applied to the pushrim with positive being inward.
Tangential force applied to the pushrim with positive resulting in forward mo- tion of the wheelchair.
Camber angle of wheel.
Misalignment angle of wheel.
Pushrim forces in the presence of cam- ber.
Pushrim forces in the presence of mis- alignment.
Pushrim forces in the presence of cam- ber and misalignment.

\section{INTRODUCTION}

$\mathbf{T}$ THERE is a growing body of literature related to the biomechanics of wheelchair propulsion [1]-[11]. Much of the wheelchair biomechanics literature is related to kinematic measurements of arm motions during wheelchair propulsion. However, recently there have been more reports of kinetic measurements of wheelchair propulsion. Unlike motion anal- 
ysis systems, kinetic measurements require the use of custom pushrim force/moment measuring instruments which are not currently commercially available. Along with the ability to measure pushrim forces and moments has come the development of several kinetic variables derived for analyzing key aspects of wheelchair propulsion.

Means of characterizing force and moment sensing systems have been published for some of the pushrim force and moment sensing systems currently in use. Rodgers et al. [12] described an instrumented pushrim used in their studies. Their studies used a $38-\mathrm{cm}$ diameter pushrim, which was specially instrumented at The Pennsylvania State University. Their system permits continuous sampling of tangential force applied to the pushrim. Sixteen strain gauges were arranged in opposing pairs on each of four pushrim supports to form a single bridge. Data were collected through a 12-bit analogto-digital converter board and software loaded on a 386 DOS compatible computer.

Niesing et al. [13] described a stationary ergometer designed for the analysis of various simulated wheelchair pushing conditions such as varying resistance, velocity, and slope. Pushrim forces were measured in three directions (tangential, radial, and axial) through transducers mounted on the axle attachment point. Data were sampled at $50 \mathrm{~Hz}$ per channel. The standard error for multiple regression with the seat and backrest sensors was found to be within $4.5 \mathrm{~N}$ over a range of $400 \mathrm{~N}$. Crosstalk of the force transducers stayed below $3 \%$ in all cases. Their device is reported to have a resolution of $1 \mathrm{~N}$ in all directions.

Strauss et al. reported on the development of a dynamic force and torque sensing wheelchair wheel [14]. The calibration of their system revealed problems in terms of linearity and drift which only permitted reliable measurement of torque. A brief description of a second prototype was reported to employ an AMTI six degrees of freedom strain gauge based force transducer, AD1B31AN strain-gauge signal conditioners, and AD8471JN amplifiers for measuring force [15]. It was stated that their system transfers data from the sensor to a computer either through a direct wire link or via a microprocessor based digital FM transmitter-receiver system.

The accuracy of pushrim force/moment measurements, and variables derived from these quantities is based upon the validity of the measurement system. Devices have been constructed to measure wheelchair propulsion forces and moments, but uncertainty associated with the measurements has not been available. The confidence in the values presented in several published reports on wheelchair propulsion biomechanics, and similarly in force plate studies, is unknown because the certainty in the measurements or measurement systems are unavailable. The uncertainty in the measurement of biomechanical variables during wheelchair propulsion depends on the configuration of the wheelchair or ergometer, the design and properties of the force/moment sensing system, and upon the forces/moments produced by the test subject. The interactions of these variables reflects the complexity of investigating wheelchair propulsion biomechanics, and warn of possible pitfalls which could lead to spurious results.

The SMART ${ }^{\text {Wheel }}$ is a pushrim force and torque sensor which was designed, fabricated, calibrated, and tested by Cooper and colleagues [9]-[11], [16], [17]. This study presents several of the key equations used to calculate or derive the variables used in the study of wheelchair propulsion biomechanics. The uncertainties for these variables were determined, and then numerically calculated for a current version of the SMART ${ }^{\text {Wheel }}$ [10], [11]. The equations and methods presented and analyzed in this paper are generalizable to other pushrim force and moment sensing systems besides the specific example presented here. Our methods are based upon approaches outlined in the ANSI/ASME Standard on Measurement Uncertainty [18]-[20]. We developed a methodology and demonstrated by example how uncertainty in measured or derived variables propagates into uncertainty of experimental and analytical results.

\section{General UnCERTAinty Framework}

Uncertainty is important when we are using experimentally determined information in an analytical solution. It is of utmost importance to consider the quality of the experimental data. Uncertainty is also important for anyone comparing results of a mathematical model with experimental data. Uncertainty is founded on the concept that collecting valid data requires meticulous planning of the experiment, and an appreciation for the measurement process. To study wheelchair biomechanics, experimental results are necessary before realistic assumptions and idealizations can be made so that mathematical or statistical models can be formulated. In addition, experimentally determined information is required in auxiliary equations necessary for obtaining answers to biomechanical or medical questions. The uncertainty analyzes used in this paper are derived from Taylor Series expansions as presented in [18]. The uncertainty $\left(U_{p}\right)$ for a data reduction function $(p)$ is found by using partial derivatives

$$
\begin{aligned}
& p= p\left(q_{1}, q_{2}, \cdots, q_{n}\right) \\
& U_{p}=\left[\left(\frac{\partial p}{\partial q_{1}} U_{q_{1}}\right)^{2}+\left(\frac{\partial p}{\partial q_{2}} U_{q_{2}}\right)^{2}\right. \\
&\left.+\cdots+\left(\frac{\partial p}{\partial q_{N}} U_{q_{n}}\right)^{2}\right]^{1 / 2}
\end{aligned}
$$

where $q_{i}$ are the experimentally measured independent variables. The uncertainty equation can be simplified by using absolute sensitivity coefficients $(\gamma)$

$$
U_{p}=\left[\sum_{i}^{n} \gamma_{i}^{2} U_{q_{i}}^{2}\right]^{1 / 2} \quad \text { where } \quad \gamma_{i}=\frac{\partial p}{\partial q_{i}} .
$$

The absolute sensitivity coefficients represent the change in the data reduction function due to changes in a variable contained within the function. This provides an indication of the contribution of the uncertainty in each independent parameter to the dependent variable. 


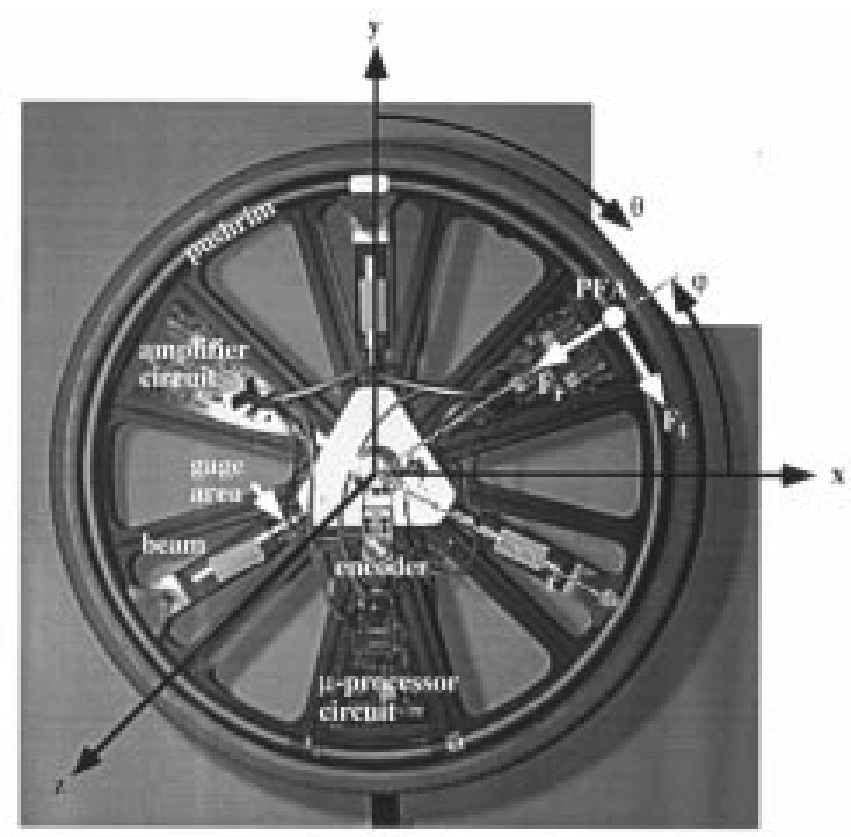

Fig. 1. Ilustration of SMART Wheel with axes, definitions, and key components identified.

\section{PUSHRIM FORCES AND MOMENTS}

The following is a simple mathematical model of the measurements necessary to calculate $F x, F y, F z, M x, M y$, and $M z$ for a six degrees of freedom force/moment recording pushrim; see Fig. 1. Channels 1-3 measure beam deflection in the plane of the wheel, and channels 4-6 measure deflection along the wheel axle. The measured signals for the six channels are referred to as $v_{i}$, while $k_{j}$ are the calibration constants for the six channels. The position of a reference beam with respect to top-dead center is given by $\theta$ where $0^{\circ} \leq \theta<360^{\circ}$. The distance between the center of the hub and the point at which the push-rim attaches to the beam is given as $r$. Assuming no crosstalk with zero camber for a fixed inertial coordinate system, the measurement equations for SMART ${ }^{\text {Wheel }}$ pushrim forces are

$F_{x}=k_{1} v_{1} \cos \theta+k_{2} v_{2} \cos \left(\theta+\frac{2 \pi}{3}\right)+k_{3} v_{3} \cos \left(\theta+\frac{4 \pi}{3}\right)$

$F_{y}=k_{1} v_{1} \sin \theta+k_{2} v_{2} \sin \left(\theta+\frac{2 \pi}{3}\right)+k_{3} v_{3} \sin \left(\theta+\frac{4 \pi}{3}\right)$

$F_{z}=k_{4} v_{4}+k_{5} v_{5}+k_{6} v_{6}$.

Measurement equations for SMART ${ }^{\text {Wheel }}$ pushrim moments are

$$
\begin{aligned}
M_{x}= & k_{4} v_{4} r \cos \theta+k_{5} v_{5} r \cos \left(\theta+\frac{2 \pi}{3}\right) \\
& +{ }_{6} v_{6} r \cos \left(\theta+\frac{4 \pi}{3}\right) \\
M_{y}= & k_{4} v_{4} r \sin \theta+k_{5} v_{5} r \sin \left(\theta+\frac{2 \pi}{3}\right) \\
& +k_{6} v_{6} r \sin \left(\theta+\frac{4 \pi}{3}\right) \\
M_{z}= & k_{1} v_{1} r+k_{2} v_{2} r+k_{3} v_{3} r .
\end{aligned}
$$

The no crosstalk assumption was validated by determining a complete set of coefficients for a voltage-force transformation matrix. Our data show that the cross-terms are less than $2 \%$ of the meaaured values for the current SMART ${ }^{\text {Wheel }}$ [11].

\section{DERIVATION OF UNCERTAINTY FOR PUSHRIM SENSING}

\section{A. Uncertainty for Pushrim Forces and Moments}

The pushrim forces are a function of the six strain gauge bridge voltages $\left(v_{i}\right)$, the six calibration coefficients $\left(k_{i}\right)$, and the pushrim angle

$F_{x, y, z}=F\left(v_{1}, v_{2}, v_{3}, v_{4}, v_{5}, v_{6}, k_{1}, k_{2}, k_{3}, k_{4}, k_{\tilde{s}}, k_{6}, \theta\right)$.

The pushrim moments are a function of the six strain gauge bridge voltages, pushrim angle, and the pushrim radius $(r)$

$$
M_{x, y, z}=M\left(v_{1}, v_{2}, v_{3}, v_{4}, v_{5}, v_{6}, k_{1}, k_{2}, k_{3}, k_{4}, k_{5}, k_{6}, \theta, r\right) .
$$

Since the functions for the pushrim forces and moments are known in closed form, the uncertainty can be calculated. The uncertainty equation for the horizontal force $\left(U_{F x}\right)$ is

$$
\begin{aligned}
U_{F_{x}}=\left[k_{1}^{2} \cos ^{2} \theta U_{v_{1}}^{2}+k_{2}^{2} \cos ^{2}\left(\theta+\frac{2 \pi}{3}\right) U_{v_{2}}^{2}\right. \\
\quad+k_{3}^{2} \cos ^{2}\left(\theta+\frac{4 \pi}{3}\right) U_{v_{3}}^{2} \\
+v_{1}^{2} \cos ^{2} \theta U_{k_{1}}^{2}+v_{2}^{2} \cos ^{2}\left(\theta+\frac{2 \pi}{3}\right) U_{k_{2}}^{2} \\
+v_{3}^{2} \cos ^{2}\left(\theta+\frac{4 \pi}{3}\right) U_{k_{3}}^{2} \\
+\left(-k_{1} v_{1} \sin \theta-k_{2} v_{2} \sin \left(\theta+\frac{2 \pi}{3}\right)\right. \\
\left.\left.\quad-k_{3} v_{3} \sin \left(\theta+\frac{4 \pi}{3}\right)\right)^{2} U_{\theta}^{2}\right]^{1 / 2}
\end{aligned}
$$

The uncertainty equation for the vertical force $\left(U_{F y}\right)$ is

$$
\begin{aligned}
U_{F_{y}}=\left[k_{1}^{2} \sin ^{2} \theta U_{v_{1}}^{2}+k_{2}^{2} \sin ^{2}\left(\theta+\frac{2 \pi}{3}\right) U_{v_{2}}^{2} /\right. \\
\quad+k_{3}^{2} \sin ^{2}\left(\theta+\frac{4 \pi}{3}\right) U_{v_{3}}^{2} \\
+v_{1}^{2} \sin ^{2} \theta U_{k_{1}}^{2}+v_{2}^{2} \sin ^{2}\left(\theta+\frac{2 \pi}{3}\right) U_{k_{2}}^{2} \\
+v_{3}^{2} \sin ^{2}\left(\theta+\frac{4 \pi}{3}\right) U_{k_{3}}^{2} \\
+\left(k_{1} v_{1} \cos \theta+k_{2} v_{2} \cos \left(\theta+\frac{2 \pi}{3}\right)\right. \\
\left.\left.\quad+k_{2} v_{3} \cos \left(\theta \frac{4 \pi}{3}\right)\right)^{2} U_{\theta}^{2}\right]^{1 / 2}
\end{aligned}
$$

The uncertainty equation for the axial force $\left(U_{F z}\right)$ is

$$
\begin{aligned}
U_{F_{z}}= & {\left[k_{4}^{2} U_{v_{4}}^{2}+k_{5}^{2} U_{v_{5}}^{2}+k_{6}^{2} U_{v_{6}}^{2}+v_{4}^{2} U_{k_{4}}^{2}\right.} \\
& \left.+v_{5}^{2} U_{k_{5}}^{2}+v_{6}^{2} U_{k_{6}}^{2}\right]^{1 / 2} .
\end{aligned}
$$


The uncertainty equation for the moment about the vertical axis $\left(U_{M x}\right)$ is

$$
\begin{aligned}
U_{M_{x}}=\left[k_{4}^{2} r^{2} \cos ^{2} \theta U_{v_{4}}^{2}+k_{5}^{2} \cos ^{2}\left(\theta+\frac{2 \pi}{3}\right) U_{v_{5}}^{2}\right. \\
+k_{6}^{2} \cos ^{2}\left(\theta+\frac{4 \pi}{3}\right) U_{v_{6}}^{2} \\
+v_{4}^{2} r^{2} \cos ^{2} \theta U_{k_{4}}^{2}+v_{5}^{2} \cos ^{2}\left(\theta+\frac{2 \pi}{3}\right) U_{k_{5}}^{2} \\
+v_{6}^{2} \cos ^{2}\left(\theta+\frac{4 \pi}{3}\right) U_{k_{6}}^{2} \\
+\left(-k_{4} v_{4} r \sin \theta-k_{5} v_{5} r \sin \left(\theta+\frac{2 \pi}{3}\right)\right. \\
\\
\left.+\quad-k_{6} v_{6} r \sin \left(\theta+\frac{4 \pi}{3}\right)\right)^{2} U_{\theta}^{2} \\
+\left(k_{4} v_{4} \cos \theta+k_{5} v_{5} \cos \left(\theta+\frac{2 \pi}{3}\right)\right. \\
\left.\left.\left.\quad+k_{6} v_{6} \cos \left(\theta+\frac{4 \pi}{3}\right)\right)^{2} U^{2}\right) r\right]^{1 / 2}
\end{aligned}
$$

The uncertainty equation for the moment about the horizontal axis $\left(U_{M y}\right)$ is

$$
\begin{aligned}
& U_{M_{y}}=\left[k_{4}^{2} r^{2} \sin ^{2} \theta U_{v_{4}}^{2}+k_{5}^{2} \sin ^{2}\left(\theta+\frac{2 \pi}{3}\right) U_{v_{5}}^{2}\right. \\
&+k_{6}^{2} \sin ^{2}\left(\theta+\frac{4 \pi}{3}\right) U_{v_{6}}^{2} \\
&+v_{4}^{2} r^{2} \sin ^{2} \theta U_{k_{4}}^{2}+v_{5}^{2} \sin ^{2}\left(\theta+\frac{2 \pi}{3}\right) U_{k_{5}}^{2} \\
&+v_{6}^{2} \sin ^{2}\left(\theta+\frac{4 \pi}{3}\right) U_{k_{6}}^{2} \\
&+v_{4}^{2} r^{2} \sin ^{2} \theta U_{k_{4}}^{2}+v_{5}^{2} \sin ^{2}\left(\theta+\frac{2 \pi}{3}\right) U_{k_{5}}^{2} \\
&+v_{6}^{2} \sin ^{2}\left(\theta+\frac{4 \pi}{3}\right) U_{k_{6}}^{2} \\
&+\left(k_{4} v_{4} r \cos \theta+k_{5} v_{5} r \cos \left(\theta+\frac{2 \pi}{3}\right)\right. \\
&\left.+k^{6} v_{6} r \cos \left(\theta+\frac{4 \pi}{3}\right)\right)^{2} U_{\theta}^{2} \\
&+\left(k_{4} v_{4} \sin \theta+k_{5} v_{5} \sin \left(\theta+\frac{2 \pi}{3}\right)\right. \\
&\left.\left.+k_{6} v_{6} \sin \left(\theta+\frac{4 \pi}{3}\right)\right)^{2} U_{r}^{2}\right]^{1 / 2} \\
&+1
\end{aligned}
$$

The uncertainty equation for the moment about the wheel axle $\left(U_{M z}\right)$ is

$$
\begin{aligned}
U_{M_{z}}=\left[k_{1}^{2} U_{v_{1}}^{2}+k_{2}^{2} U_{v_{2}}^{2}+k_{3}^{2} U_{v_{3}}^{2}+v_{1}^{2} U_{k_{1}}^{2}\right. \\
\left.+v_{2}^{2} U_{k_{2}}^{2}+v_{3}^{2} U_{k_{3}}^{2}\right]^{1 / 2} .
\end{aligned}
$$

\section{B. Uncertainty for Point of Force Application}

The point of force application is a virtual location on the pushrim [16]; see Fig. 1. The point of force application (PFA) is determined from the forces and moments applied to the pushrim. By definition, the PFA must lie on the pushrim. Therefore, the PFA can be defined in Cartesian coordinates, $\left(x_{\mathrm{PFA}}, y_{\mathrm{PFA}}, z_{\mathrm{PFA}}\right)$ with the origin at the wheel hub as $(r \cos \varphi, r \sin \varphi, 0)$ where $\varphi$ is defined with respect to the horizontal. The point of force application angle $(\varphi)$ can be determined, with a six degrees of freedom system, using the pushrim moments about the $x$ and $y$ axis: $M_{x}$ and $M_{y}$, respectively

$$
\begin{aligned}
M_{y} & =F_{z} R \cos \varphi+F_{x} z+m_{y} \\
& =F_{z} x_{\mathrm{PFA}}+F_{x} z_{\mathrm{PFA}}+m_{y} \\
M_{x} & =F_{z} R \sin \varphi+F_{y} z+m_{x} \\
& =F_{z} y_{\mathrm{PFA}}+F_{x} z_{\mathrm{PFA}}+m_{x} .
\end{aligned}
$$

By definition $z_{\mathrm{PFA}}$ is zero for all time during the propulsion and recovery phases of the stroke. This is due to the point of force application being forced to be on the pushrim. Based on this definition, (13) reduces to (14)

$$
\tan \varphi=\frac{M_{x}-m_{x}}{M_{y}-m_{y}} .
$$

Equation (14) shows that the PFA is dependent upon the wrench moments applied by the wrist to the pushrim about the absolute $x$ and $y$ axes: $m_{x}$ and $m_{y}$, respectively. The wrist moment components are additive terms in the pushrim moment equations. The uncertainty of the pushrim point of force application $\left(U_{\varphi}\right)$ described in $(15)$ is given by

$$
\begin{aligned}
U_{\varphi}=[( & \left.\frac{\partial \varphi}{\partial M_{x}} U_{M_{x}}\right)^{2}+\left(\frac{\partial \varphi}{\partial m_{x}} U_{m_{x}}\right)^{2} \\
& \left.+\left(\frac{\partial \varphi}{\partial M_{y}} U_{M_{y}}\right)^{2}+\left(\frac{\partial \varphi}{\partial m_{y}}\right)^{2}\right]^{1 / 2} \\
U_{\varphi}=\left[\left(\frac{\left(M_{y}-m_{y}\right)}{\left(M_{x}-m_{x}\right)^{2}+\left(m_{y}-m_{y}\right)^{2}} U_{M_{x}}\right)^{2}\right. & +\left(\frac{-\left(M_{y}-m_{y}\right)}{\left(M_{x}-m_{x}\right)^{2}+\left(M_{y}-m_{y}\right)^{2}} U_{m_{x}}\right)^{2} \\
& +\left(\frac{-\left(M_{x}-m_{x}\right)}{\left(M_{x}-m_{x}\right)^{2}+\left(M_{y}-m_{y}\right)^{2}} U_{M_{y}}\right)^{2} \\
& \left.+\left(\frac{\left(M_{x}-m_{x}\right)}{\left(M_{x}-m_{x}\right)^{2}+\left(M_{y}-m_{y}\right)^{2}} U_{m_{y}}\right)^{2}\right]^{1 / 2} .
\end{aligned}
$$

\section{Uncertainty for Pushrim Radial and Tangential Forces}

Radial and tangential forces are not currently measured directly with the SMART ${ }^{\text {Wheel }}$. They are derived variables which depend upon the absolute forces and the point of force application. The radial and tangential forces are dependent upon the point of force application, and the forces applied 
to the pushrim without camber or misalignment are

$$
\left[\begin{array}{l}
F_{r} \\
F_{t} \\
F_{z}
\end{array}\right]=\left[\begin{array}{ccc}
-\cos \varphi & -\sin \varphi & 0 \\
\sin \varphi & -\cos \varphi & 0 \\
0 & 0 & 1
\end{array}\right]\left[\begin{array}{l}
F_{x} \\
F_{y} \\
F_{z}
\end{array}\right] .
$$

The uncertainty for the radial force is a function of the point of force application, as well as the $x$ and $y$ forces, are

$$
\begin{aligned}
U_{F_{r}}= & {\left[\cos ^{2} \varphi U_{F_{x}}^{2}+\sin ^{2} \varphi U_{F_{y}}^{2}\right.} \\
& \left.+\left(F_{x} \sin \varphi-F_{y} \cos \varphi\right)^{2} U_{\varphi}^{2}\right]^{1 / 2} .
\end{aligned}
$$

The uncertainty for the tangential force is, similarly, a function of the point of force application, as well as the $x$ and $y$ forces

$$
\begin{aligned}
U_{F_{t}}=[ & \sin ^{2} \varphi U_{F_{x}}^{2}+\cos ^{2} \varphi U_{F_{y}}^{2} \\
& \left.+\left(F_{x} \cos \varphi-F_{y} \sin \varphi\right)^{2} U_{\varphi}^{2}\right]^{1 / 2} .
\end{aligned}
$$

\section{Pushrim Force Uncertainty with Camber and Misalignment}

The pushrim radial and tangential forces are calculated assuming that camber is set first, then the alignment is corrected, and finally we account for the rotation of the point of force application. The camber angle, i.e., rotation about the global $x$ axis, can be accommodated using the following homogeneous transformation matrix:

$$
\left[\begin{array}{l}
F_{x} \\
F_{y} \\
F_{z}
\end{array}\right]=\left[\begin{array}{ccc}
1 & 0 & 0 \\
0 & \cos \beta & \sin \beta \\
0 & -\sin \beta & \cos \beta
\end{array}\right]\left[\begin{array}{l}
F_{x_{c}} \\
F_{y_{c}} \\
F_{z_{c}}
\end{array}\right] .
$$

The SMART ${ }^{\text {Wheel }}$ measures forces in the local coordinate space. Therefore, once camber is added the measured forces must be transformed into the global coordinate space $(x, y, z)$. Wheel alignment, i.e., rotation about the global $y$-axis, may change after camber is adjusted. If this occurs, then the measured forces will again need to be transformed into the global coordinate space

$$
\left[\begin{array}{l}
F_{x} \\
F_{y} \\
F_{z}
\end{array}\right]=\left[\begin{array}{ccc}
\cos \alpha & 0 & -\sin \alpha \\
0 & 1 & 0 \\
\sin \alpha & 0 & \cos \alpha
\end{array}\right]\left[\begin{array}{l}
F_{x_{a}} \\
F_{y_{a}} \\
F_{z_{a}}
\end{array}\right] .
$$

The pushrim force and moment equations in the previous sections, are independent of camber and alignment as they are recorded relative to the pushrim. If there is camber and or wheel misalignment, then the forces and moments at the pushrim in the global coordinate frame become

$$
\begin{aligned}
{\left[\begin{array}{l}
F_{x} \\
F_{y} \\
F_{z}
\end{array}\right]=} & {\left[\begin{array}{ccc}
\cos \alpha & 0 & -\sin \alpha \\
0 & 1 & 0 \\
\sin \alpha & 0 & \cos \alpha
\end{array}\right]\left[\begin{array}{ccc}
1 & 0 & 0 \\
0 & \cos \beta & \sin \beta \\
0 & -\sin \beta & \cos \beta
\end{array}\right] } \\
& \cdot\left[\begin{array}{l}
F_{x_{c, a}} \\
F_{y_{c, a}} \\
F_{z_{c, a}}
\end{array}\right] \\
{\left[\begin{array}{l}
F_{x} \\
F_{y} \\
F_{z}
\end{array}\right]=} & {\left[\begin{array}{ccc}
\cos \alpha & \sin \alpha \sin \beta & -\sin \alpha \cos \beta \\
0 & \cos \beta & \sin \beta \\
\sin \alpha & -\cos \alpha \sin \beta & \cos \alpha \cos \beta
\end{array}\right] } \\
& \cdot\left[\begin{array}{l}
F_{x_{c, a}} \\
F_{y_{c, a}} \\
F_{z_{c, a}}
\end{array}\right] .
\end{aligned}
$$

The pushrim forces and moments in the global coordinate frame are in a form which can be used to determine their uncertainty

$F_{x}=F_{x_{c, a}} \cos \alpha+F_{y_{c, a}} \sin \alpha \sin \beta-F_{z_{c, a}} \sin \alpha \cos \beta$

$F_{y}=F_{y_{c, a}} \cos \beta+F_{z_{c, a}} \sin \beta$

$F_{z}=F_{x_{c, a}} \sin \alpha-F_{y_{c, a}} \cos \alpha \sin \beta+F_{z_{c, a}} \cos \alpha \cos \beta$.

The uncertainty for the horizontal force in the global coordinate frame is

$$
\begin{aligned}
U_{F_{x}}=\left[\cos ^{2} \alpha U_{F_{x_{c}, a}}^{2}+\sin ^{2} \alpha \sin ^{2} \beta U_{F_{y_{c}, a}}^{2}\right. & \\
& +\sin ^{2} \alpha \cos ^{2} \beta U_{F_{z_{c}, a}}^{2} \\
& +\left(-F_{x_{c, a}} \sin \alpha+F_{y_{c, a}} \cos \alpha \sin \beta\right. \\
& \left.\quad-F_{z_{c, a}} \cos \alpha \cos \beta\right)^{2} U_{\alpha}^{2} \\
& \left.+\left(F_{y_{c, a}} \sin \alpha \cos \beta+F_{z_{c, a}} \sin \alpha \sin \beta\right)^{2} U_{\beta}^{2}\right]^{1 / 2} .
\end{aligned}
$$

The uncertainty for the vertical force in the global coordinate frame is

$$
\begin{aligned}
U_{F_{y}}=\left[\cos ^{2} \beta U_{F_{y_{c}, a}}^{2}+\sin ^{2} \beta U_{F_{z_{c}, a}}^{2}\right. & \\
& \left.+\left(-F_{y_{c, a}} \sin \beta+F_{z_{c, a}} \cos \beta\right)^{2} U_{\beta}^{2}\right]^{1 / 2} .
\end{aligned}
$$

The uncertainty for the axial force in the global coordinate frame is

$$
\begin{aligned}
U_{F_{z}}=\left[\sin ^{2} \alpha U_{F_{x_{c}, a}}^{2}+\cos ^{2} \alpha \sin ^{2} \beta U_{F_{y_{c}, a}}^{2}\right. & \\
& +\cos ^{2} \alpha \cos ^{2} \beta U_{F_{z_{c}, a}}^{2} \\
& +\left(F_{x_{c, a}} \cos \alpha+F_{y_{c, a}} \sin \alpha \sin \beta\right. \\
& \left.\quad-F_{z_{c, a}} \sin \alpha \cos \right)^{2} U_{\alpha}^{2} \\
& \left.+\left(F_{y_{c, a}} \cos \alpha \cos \beta+F_{z_{c, a}} \cos \alpha \sin \beta\right)^{2} U_{\beta}^{2}\right]^{1 / 2} .
\end{aligned}
$$

\section{EXPERIMENTAL ANALYSIS}

\section{A. Experimental Protocol}

For the purpose of this study, we had a single experienced male wheelchair user with a T7-8 spinal cord injury give written informed consent and push his personal wheelchair mounted with the SMART ${ }^{\text {Wheel }}$ on a wheelchair dynamometer for as fast as he could push over a period of one minute. The resistance of the dynamometer was set to simulate a common tile floor. The wheel position, wheel alignment, and wheel camber were the same as those for the subject's standard wheelchair wheels. The subjects was asked to propel his wheelchair on the dynamometer for five minutes to become accommodated to the experimental set-up. Data were collected for the last 30 seconds of the minute.

Pushrim force/moment data were collected from the SMART $^{\text {Wheel }}$ at $240 \mathrm{~Hz}$ and low-pass filtered at $30 \mathrm{~Hz}$ [17]. The kinetic data filter was an eighth-order zero-phase digital Butterworth type. Ten point data extrapolation was used to reduce end-point anomolies from digital filtering. Phase shift was eliminated by running the data through the digital filter 


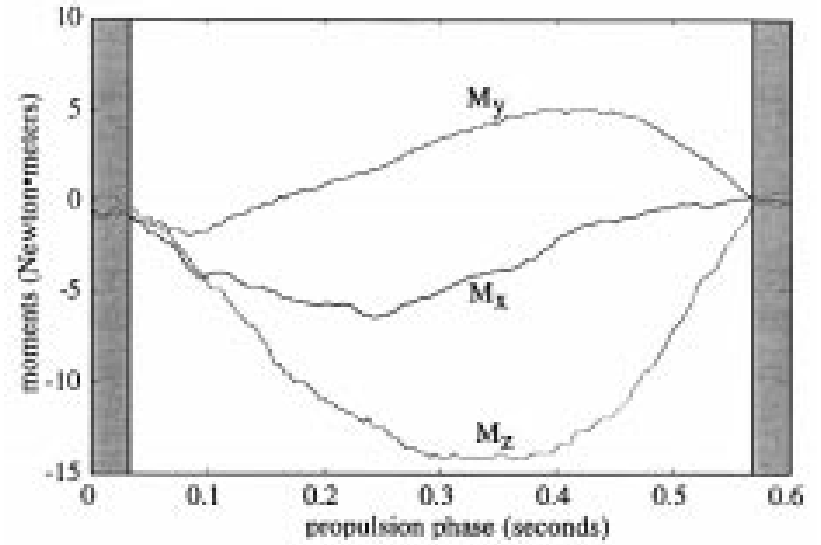

Fig. 2. Three-dimensional pushrim moments for a subject propelling his own wheelchair with the SMART ${ }^{\text {Wheel }}$. The gray bars represent the regions of high uncertainty for the point of force application.

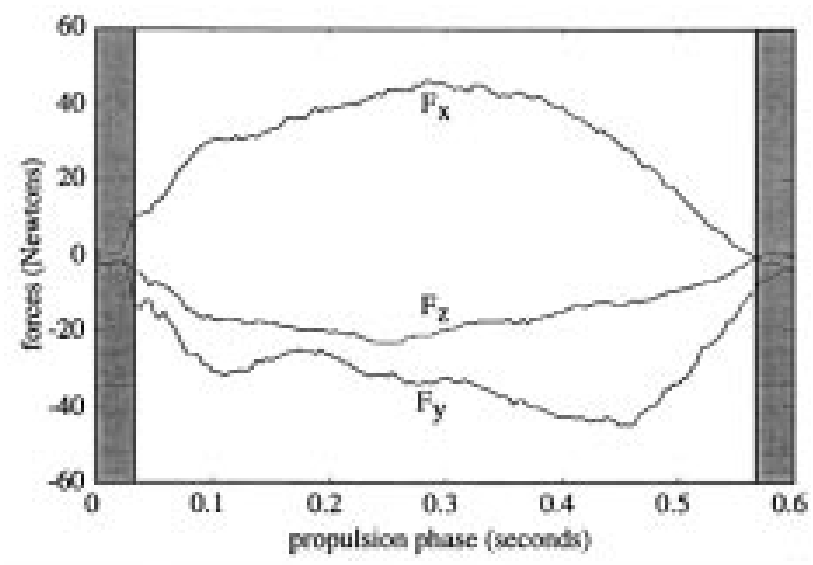

Fig. 3. Three-dimensional pushrim forces for a subject propelling his own wheelchair with the SMART Wheel. The gray bars represent the regions of high uncertainty for the radial and tangential forces.

in both the forward and reverse directions. For the purpose of this study, the propulsion phase was defined as the period when the moment about the hub $M_{z}$ deviated more than 5\% from baseline, until it once again returned to baseline and remained within 5\%. During this experiment, two standard polished and annodized aluminum pushrims of 0.2667 meters radius were mounted to the wheels. Standard gray rubber tires which were $61 \mathrm{~cm}$ (i.e., 24 in) diameter with width $3.5 \mathrm{~cm}$ (i.e., $1.375 \mathrm{in}$ ). A foam insert was used in place of a pneumatic tube. The time series curves for the moments used in this study are presented Fig. 2. The horizontal and vertical forces data collected during this study are presented in Fig. 3.

\section{B. Instrumentation}

We previously described the current three-dimensional (3-D) versions of a force and torque sensing SMART ${ }^{\text {Wheel }}$ [9], [11]. The SMART ${ }^{\text {Wheel }}$ mounts to most standard wheelchairs, and with calibration properties presented in Table I.
TABLE I

PROPERTIES OF THE LATEST VERSION OF THE 3-D SMART ${ }^{\text {Wheel }}$

\begin{tabular}{|c|c|c|c|c|}
\hline Property & Forces & Moments & Wheel Angle & $\begin{array}{l}\begin{array}{l}\text { Nalural } \\
\text { Frequency }\end{array} \\
\end{array}$ \\
\hline Percent Lincarity & $98.9 \%$ & $99.1 \%$ & $\mathrm{~N} / \mathrm{A}$ & N/A \\
\hline Range & $\pm 155 \mathrm{~N}$ & $\pm 77 \mathrm{~N} \bullet \mathrm{m}$ & $0-360$ degrees & $\mathrm{N} / \mathrm{A}$ \\
\hline Precision & $1.2 \mathrm{~N}$ & $0.6 \mathrm{~N} \cdot \mathrm{m}$ & 0.36 degrees & $\mathrm{N} / \mathrm{A}$ \\
\hline Resolution & $1.7 \mathrm{~N}$ & $1 \mathrm{~N} \cdot \mathrm{m}$ & 0.36 degrees & N/A \\
\hline $\begin{array}{l}\text { Independent } \\
\text { Non-linearity }\end{array}$ & $1.1 \%$ & $0.9 \%$ & N/A & $\mathrm{N} / \mathrm{A}$ \\
\hline Sagittal Plane & $\begin{array}{l}\text { N/A } \\
\text { N/A }\end{array}$ & N/A & N/A & $\begin{array}{l}150 \mathrm{~Hz} \\
100 \mathrm{~Hz}\end{array}$ \\
\hline
\end{tabular}

The values presented in Table I, were determined using standard calibration procedures outlined in [10] and [18]. The SMART Wheel was made by modifying a Quickie composite wheel (Sunrise Medical Incorporated) which consists of 12 pie-shaped sections; see Fig. 1. Linear bearings are used so that force applied axially to the end of a beam (toward the hub) is transferred to the other two beams. A rotary bearing assembly is attached to the end of each beam so that each beam detects only pure bending. Each beam is secured at the wheel hub via a specially designed aluminum base assembly and via pushrim stand-offs, comparable to those used on a common wheelchair wheel. All beams are instrumented with two sets of full strain gauge bridges (Micro-Measurements, EA-13-062AQ-350, $350 \mathrm{ohm}$ foil strain gauges) with d.c. excitation. Signal conditioning, filtering, and amplification for each channel are performed on the wheel. This improves the integrity of the data during transmission to the computer. An AD1B31AN strain gauge signal conditioner is used to provide the excitation voltage to the strain gauge bridge, balance the bridge, amplify, and pre-filter the signal. A six-channel mercury slip-ring is used for signal transmission off the rotating wheel. A mercury slip-ring was selected over telemetry to minimize noise interference due to other laboratory test equipment. The SMART ${ }^{\text {Wheel }}$ uses a Motorola 6811 microcontroller to perform time division multiplexing and to interface to DOS compatible personal computer. The MC6811C11A1 microcontroller has 8-bit analog-to-digital conversion on-chip and is interfaced to a standard RS232 serial computer port. The digital SMART ${ }^{\text {Wheel }}$ exhibits several properties: low drift, high fidelity, high reliability, good angular resolution (full quadrature implementation yields 0.2-degree resolution), fast response (the microcontroller is more efficient at $\mathrm{A} / \mathrm{D}$ conversion than most PC-based DACA boards), and low-power consumption [9].

\section{Performance Criteria}

During the design of the SMART ${ }^{\text {Wheel }}$, it was desired to obtain an uncertainty of less than 5\% of each variable's fullscale value during the mid-range of the propulsion phase (i.e., the area between the gray bars on Figs. 2 and 3). Previous experience with force platforms and earlier versions of the $\mathrm{SMART}^{\text {Wheel }}$ indicated this may be unobtainable during the initial and final 5\% of the propulsion phase for variables with force or moment terms in the denominators of their data reduction equations. For the purposes of this study, acceptable 
TABLE II

Basic Uncertainty VAlues USED In this Study

\begin{tabular}{l|c|c|c|c}
\hline Uncertainties & $U_{v_{i}}$ & $U_{\theta}$ & $U_{r}$ & $U_{k_{i}}$ \\
\hline Value Used & 0.02 volts & 0.36 degrees & $0.8 \mathrm{~mm}$ & $0.1 \mathrm{~N} / \mathrm{v}$ \\
\hline
\end{tabular}

TABLE III

FORCE AND MOMENT UNCERTAINTY VALUES FOR SMART Wheel

\begin{tabular}{|c|c|c|c|c|c|c|}
\hline & $U_{F_{x}}{ }^{*}$ & $U_{F_{y}}{ }^{*}$ & $U_{F_{z}}{ }^{*}$ & $U_{M_{x}} \S$ & $U_{M_{y}} \S$ & $U_{M_{z}} \S$ \\
\hline maximum & 2.5 & 2.5 & 0.93 & 0.19 & 0.19 & 2.24 \\
\hline minimum & 1.1 & 1.1 & 0.93 & 0.03 & 0.03 & 2.24 \\
\hline $\begin{array}{l}\text { root-mean- } \\
\text { square }\end{array}$ & 2.2 & 2.2 & 0.93 & 0.09 & 0.09 & 2.24 \\
\hline
\end{tabular}

performance for the uncertainty for the $x, y, z$ forces and moments (shown in Table III) was defined to be less than two times the resolution; see Table I.

\section{RESUltS AND SimULATIONS}

\section{A. Basic Variable Uncertainties}

The uncertainty in the measurements of each of the voltages are equal

$$
U_{v_{1}}=U_{v_{2}}=U_{v_{3}}=U_{v_{4}}=U_{v_{5}}=U_{v_{6}}
$$

for the current SMART ${ }^{\text {Wheel }}$ system. The voltage uncertainty for the system is about $0.02 \mathrm{~V}$, which is about one bit of a 256bit analog to digital converter with range of $5 \mathrm{~V}$. The strain gauge amplifier gains are tuned to take advantage of the entire five volts range without clipping. The angle uncertainty for $q$ is about $0.36^{\circ}$ [9], [11]. The pushrim rotates a full $360^{\circ}$, therefore $q$ varies from 0 to $360^{\circ}$. The radius uncertainty is dependent upon the accuracy with which the radius can be made and measured. We were capable of measuring the pushrim radius with a machinist caliper to within $0.8 \mathrm{~mm}$. The uncertainties for the calibration constants were defined as the mean of the standard deviations of the $k_{i}$ 's from the results of repeating the calibration procedures three times. Table II presents the basic uncertainties used in this study.

\section{B. Force and Moment Uncertainty for SMART Wheel}

Because of the functional dependency of the force and moment uncertainties on the voltage, calibration constant, angle and radius, a range of values results. The minimum and maximum uncertainties were determined numerically for one full revolution of the wheel; see Table III.

The force and moment uncertainties form the foundation for the other important biomechanical variables which are derived from the measured forces and moments. The uncertainties for these derived variables will be examined more closely in the following sections.
TABLE IV

UNCERTAINTIES FOR RADIAL AND TANGENTIAL FORCES

\begin{tabular}{l|c|c|c|c}
\hline & \multicolumn{2}{|c|}{$\begin{array}{l}U_{F_{r}} \\
\text { (Ncwtons) }\end{array}$} & \multicolumn{2}{c}{$\begin{array}{c}U_{F_{t}} \\
\text { (Newtons) }\end{array}$} \\
\hline & $U_{\varphi_{\max }}$ & $U_{\varphi_{\text {rms }}}$ & $U_{\varphi_{\max }}$ & $U_{\varphi_{r m s}}$ \\
\hline $\begin{array}{l}\text { maximum } \\
\text { root-mean- } \\
\text { squarc }\end{array}$ & 13.4 & 2.9 & 19.4 & 3.4 \\
\hline minimum & 9.4 & 2.7 & 12.6 & 2.9 \\
\hline
\end{tabular}

\section{Point of Force Application Uncertainty}

The uncertainty for the point of force application (PFA) was shown to be dependent upon the uncertainty for the moments about the $\mathrm{x}$ and $\mathrm{y}$ axes, as well as the values of the moments themselves. The sensitivity of the PFA to small forces and moments is illustrated in the uncertainty analysis results. The PFA uncertainty was calculated using the maximum values for the uncertainties for the pushrim moments. When the moments are small, within the first and final 5\% of the propulsion phase, the uncertainty of the PFA is $360^{\circ}$. Which means that the location of the point of force application can lie anywhere on the pushrim during this portion of the stroke. When the moments are larger, in the middle $80 \%$ of the propulsion phase, the PFA uncertainty has a maximum value of $16^{\circ}$, a minimum value of $0.6^{\circ}$, and a root mean square value of $1.8^{\circ}$. Our data indicate that the SMART ${ }^{\text {Wheel }}$ can detect the PFA position to within $1 \mathrm{~cm}$ when the moments are greater than $2 \mathrm{~N} \cdot \mathrm{m}$.

\section{Radial and Tangential Force Uncertainty}

The uncertainties for the radial and tangential pushrim forces are functions of the point of forces application, the horizontal force, the vertical force, and their respective uncertainties. The range of uncertainties for the radial and tangential forces were calculated numerically using the maximum values for the uncertainties of the variables upon which they depend. The horizontal and vertical forces corresponding to the moment data in Fig. 2 were used to calculate the uncertainties for the radial and tangential forces; see Fig. 3. The range of the calculated PFA values for the same stroke were also used in the calculations.

Two values of PFA uncertainty were used: the maximum value during the mid-range of the propulsion phase and the root mean square (rms) value of the uncertainty during the midrange of the propulsion phase. Midrange is defined as the region between the gray bars on Figs. 2 and 3. The results for the radial and tangential force uncertainty analysis are presented in Table IV.

\section{E. Effects of Wheel Alignment and Camber on Pushrim Force Uncertainty}

The effect of wheel misalignment and camber on the horizontal, vertical, and axial force components are shown in Table V. For these calculations, the maximum uncertainties for the forces were used from Table III. The forces used to determine these uncertainties are those shown in Fig. 3. 
TABLE V

UNCERTAINTIES IN THE PUSHRIM FORCES with Wheel CAmber and Misalignment

\begin{tabular}{c|c|c|c}
\hline & $\begin{array}{c}U_{F_{x}} \\
\text { (Newtons) }\end{array}$ & $\begin{array}{c}U_{F_{y}} \\
\text { (Newtons) }\end{array}$ & $\begin{array}{c}U_{F_{z}} \\
\text { (Newtons) }\end{array}$ \\
\hline maximum & 2.5 & 2.5 & 0.5 \\
\hline root-mean-squarc & 2.5 & 2.5 & 0.5 \\
\hline minimum & 2.5 & 2.5 & 0.4 \\
\hline
\end{tabular}

Misalignment and camber have the effect of increasing the uncertainty for all of forces. The percent increase is similar in all directions. For these calculations, it was assumed that both camber and misalignment were measured to within $4 \mathrm{~mm}$ over the diameter of a standard wheelchair wheel (24 in: $0.61 \mathrm{~m})$. Based upon our experience, this is a conservative estimate. The misalignment angle was assumed to be centered around the ideal alignment of $0^{\circ}$. The camber angle was assumed to be centered around the common wheelchair camber angle of $4^{\circ}$.

\section{DISCUSSION}

The uncertainty analysis for the SMART ${ }^{\text {Wheel }}$ shows that although the calibration results are acceptable, there are interactions within the data reduction equations taking place to which investigators must be aware. This is not to say that the SMART ${ }^{\text {Wheel }}$ does not provide accurate or useful data; rather, the uncertainty analysis provides a more complete picture of the attainable accuracy of the SMART ${ }^{\text {Wheel }}$, and the degree of confidence with which the data can be recorded. In most cases, we have presented conservative estimates of the uncertainty with the current SMART ${ }^{\text {Wheel }}$. This was accomplished by using extreme values in many cases. The uncertainty results indicate that the SMART ${ }^{\text {Wheel }}$ provides data which has better than 5-10\% accuracy over the entire propulsion phase, depending upon the variable concerned. During mid-range of the propulsion phase the uncertainty is considerably smaller (i.e., approximately $1 \%$ ).

The derivations of the uncertainty equations for wheelchair propulsion biomechanics make two other notable contributions: the equations and results may be used to determine the statistical power required to detect differences between groups or between an individual in response to changes in the wheelchair; and the derivations and results indicate where improvements to measurement of wheelchair propulsion biomechanical variables are likely to originate. The former contribution relates to the variance in the wheelchair propulsion biomechanical variables due to inherent uncertainty in the entire system. Therefore, any differences between groups or interventions must rely on variations which exceed the uncertainty in the biomechanical variable. The latter contribution addresses the issue of how does one improve the measurements (i.e., reduce the uncertainty). The most efficient approach is to address those variables in the design of the system which make the greatest contribution to the uncertainty.

The methods presented in this paper and the corresponding results provide some guidance for experiment design for wheelchair propulsion studies. The data reduction equations for the biomechanical variables presented in this paper, and their corresponding uncertainty equations are similar to those used in nearly all published reports on wheelchair propulsion kinetics. Investigators may apply the information presented in this paper to their systems, by substituting their basic uncertainty values into the equations presented in this paper. When designing a biomechanical experiment with uncertainties, the protocol must elicit sufficiently large changes in the variables of interest or the uncertainties in the instrumentation or data reduction equations must be reduced. Pilot data should be used when designing experiments to determine if the intervention or condition induces differences greater than the uncertainty.

The point of force application provides the greatest source of uncertainty. Further research should focus on reducing this uncertainty. This may be accomplished through improving the ability to record moments and/or by developing more robust data reduction equations for determining the PFA. The point of force application is an important variable when studying wheelchair propulsion biomechanics. The PFA is used as the starting point for calculating net joint moments and forces using the inverse Euler method [7], [8]. Variations in the PFA may also be used to discriminate between propulsion techniques from differing groups. However, the PFA is illconditioned, because of the $\left(M_{x}^{2}-m_{x}^{2}\right)$ and $\left(M_{y}^{2}-m_{y}^{2}\right)$ components in the denominators, when the pushrim moments are small. Previously, investigators have visually inspected graphs of the PFA to determine when its was suitably stable. The uncertainty of the PFA presented in (15), demonstrates why the variation in the PFA occurs with small moments, and provides an analytical means of determining when the PFA is suitably stable. This can be accomplished by setting an acceptable limit on the PFA uncertainty.

The uncertainties for the radial and tangential forces demonstrate higher peak values, than do the horizontal and vertical forces. The increased uncertainty is due to the component attributable to the uncertainty in the PFA. Therefore, techniques which would improve the measurement of PFA location, would also have a positive impact on the radial and tangential force measurement. Wheel camber and alignment also influence the uncertainty of the force measurements. However, their effect on the horizontal and vertical force uncertainties is small. Moreover, camber angle and wheel alignment can often be treated as fixed points which would have lessor effect on the variability between groups of subjects.

Future studies will work to reduce the dependence on the no-crosstalk assumption, and on the Taylor series expansion for determining uncertainty. Biomechanical studies rely on numerical calculations. Uncertainty can be reduced by engineering numerical solutions to data reduction equations which are less sensitive to parameter variations or error propagation. Restructuring some data reduction equations or alternative solutions should be developed to reduce uncertainty in all of the variables presented in this paper. Greater understanding of the uncertainties and their influence on experiment design may be learned by taking a stochastic processes approach to error propagation and determination of confidence intervals. The 
Taylor-series approach taken in this paper, though appropriate, involves linearization which can breakdown in regions where the data reduction equation of interest is highly nonlinear. Simulations letting independent variables be Gaussian random variables with their means at the "true" values and standard deviations varied appropriately may provide greater insight into the nonlinear behavior of the data reduction equations. The means and standard deviations of the dependent variables could be used to estimate their uncertainties. This approach will be the topic of future research.

\section{REFERENCES}

[1] R. A. Cooper, D. M. Brienza, and C. E. Brubaker, "Wheelchairs and seating," Current Opinion in Orthoped., vol. 5, no. 6, pp. 101-107, 1994.

[2] S. J. Mulroy, J. K. Gronley, C.J. Newsam, and J. Perry, "Electromyographic activity of shoulder muscles during wheelchair propulsion in paraplegic persons," Archives Physic. Med. Rehab., vol. 77, pp. 187-193, 1996.

[3] F.C. Su, L.T. Lin; H.W. Wu, Y. L. Chou, K. A. A. Westreich, "A three-dimensional dynamic analysis of wheelchair propulsion," Chinese J. Med. Biologic. Eng., vol. 13, no. 4, pp. 326-342, 1993.

[4] H. E. J. Veeger, L. H. V. van der Woude, and R. H. Rozendal, "The effect of rear wheel camber in manual wheelchair propulsion," J. Rehab. Res., vol. 26, no. 2, pp. 37-46, 1989.

[5] _ "Wheelchair propulsion technique at different speeds," Scand. J. Rehab. Med., vol. 21, pp. 197-203, 1989.

[6] L. H. V. van der Woude, H. E. J. Veeger, and R. H. Rozendal, "Propulsion technique in hand rim wheelchair ambulation," J. Med. Eng. Tech., vol. 13, nos. 1 and 2, pp. 136-141, 1989.

[7] R. N. Robertson, M. L. Boninger, R. A. Cooper, and S. Shimada, "Pushrim forces and joint kinetics during wheelchair propulsion," Archives Physic. Med. Rehab., vol. 77, pp. 856-864, 1996.

[8] R. A. Cooper, Rehabilitation Engineering Applied to Mobility and Manipulation. London, England: Institute of Physics Publishing, 1995.

[9] D. P. VanSickle, R. A. Cooper, and R. N. Robertson, "SMART"WHEEL: Development of a digital force and moment sensing pushrim," in Proc. 18th Annu. RESNA Conf., Vancouver, B.C., Canada, 1995, pp. 352-354.

[10] K. T. Asato, R. A. Cooper, R. N. Robertson, and J. F. Ster, "SMART Wheels: Development and testing of a system for measuring manual wheelchair propulsion dynamics," IEEE Trans. Biomed. Eng., vol. 40, pp. 1320-1324, Dec. 1993.

[11] R. A. Cooper, D. P. VanSickle, R. N. Robertson, M. L. Boninger, and G. Ensminger, "Center of pressure during manual wheelchair propulsion," IEEE Trans. Rehab. Eng., vol. 3, pp. 289-298, Dec. 1995.

[12] M. M. Rodgers, G. W. Gayle, S. F. Figoni, M. Kobayashi, J. Lieh, and R. M. Glaser, "Biomechanics of wheelchair propulsion during fatigue," Archives Phys. Med. Rehab., vol. 75, pp. 85-93, 1994.

[13] R. Niesing, F. Eijskoot, R. Kranse, A. H. den Ouden, J. Storm, H. E. J. Veeger, L. H. V. van der Woude, and C. J. Snijders, "Computer controlled wheelchair ergometer," Med. Biol. Eng. Comput., vol. 28, pp. 329-338, 1990

[14] M. G. Strauss, M. H. Moeinzadeh, M. Schneller, and J. Trimble, "The development of an instrumented wheel to determine the handrim forces during wheelchair propulsion," in Proc. ASME Winter Annu. Meeting, 1989 , pp. 53-54.

[15] M. G. Strauss, J. Maloney, F. Ngo , and M. Phillips, "Measurement of the dynamic forces during manual wheelchair propulsion," in Proc. Amer. Soc. Biomechan. 15th Annu. Meeting, 1991, pp. 210-211.

[16] R. A. Cooper, R. N. Robertson, D. P. VanSickle, M. L. Boninger, and S. D. Shimada, "Projection of the point of force application onto the palmar surface of the hand during wheelchair propulsion," IEEE Trans. Rehab. Eng., vol. 4, pp. 133-142, Sept. 1996.

[17] R. A. Cooper, C. P. DiGiovine, M. L. Boninger, S. D.Shimada, and R. N. Robertson, "Frequency analysis of 3-dimensional pushrim forces and moments for manual wheelchair propulsion," Automedica, to be published.

[18] H. W. Coleman and W. G. Steele, Experimentation and Uncertainty Analysis for Engineers.New York: Wiley, 1989.

[19] R. J. Moffat, "Describing the uncertainties in experimental results," Experimental Thermal and Fluid Sci., vol. 1, no. 1, pp. 3-17, 1988

[20] Measurement Uncertainty. ANSI/ASME PTC 19.1-1985 Part 1, 1986.

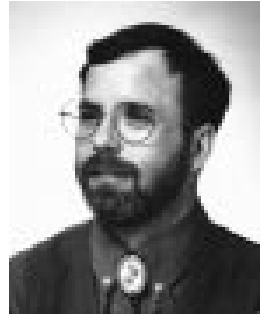

Rory A. Cooper (S'82-M'85-SM'92) received the B.S. and M.Eng degrees in electrical engineering from California Polytechnic State University, San Luis Obispo, in 1985 and 1986, respectively. He received the Ph.D. degree in electrical and computer engineering with a concentration in biomedical engineering from University of California at Santa Barbara in 1989 .

$\mathrm{He}$ is Chairman of the Department of Rehabilitation Science and Technology, and Associate Professor of Bioengineering and Mechanical Engineering at the University of Pittsburgh. He is also an Associate Professor in the Division of Physical Medicine and Rehabilitation within the Department of Orthopaedic Surgery at the University of Pittsburgh Medical Center. He is also Director of the Pitt/VAMC Human Engineering Research Laboratories which is a joint effort between the Highland Drive VAMC, University of Pittsburgh, and University of Pittsburgh Medical Center. Prior to coming to Pittsburgh, he was an Associate Professor of Biomedical Engineering and Electrical Engineering at California State University, Sacramento, where he was Coordinator of the Rehabilitation Engineering Program. He maintains relationships with several companies in the areas of rehabilitation product design, and has developed an ANSI-ISO Wheelchair Test Center. He has authored or co-authored more than one hundred papers, expanded abstracts, and book chapters. He is the author of the recent book Rehabilitation Engineering Applied to Mobility and Manipulation.

Dr. Cooper is on the Editorial Board of the VA Journal of Rehabilitation Research \& Development, the Journal of the Joint Center on Research and Prosthetics and Orthotics (Saudi Arabia), and of Team Rehab Report. He was a founding Associate Editor of the IEEE TRAnSACtions of ReHABilitation ENGINEERING. In 1992, he received the CSUS Outstanding Research Award. In 1993, he was the recipient of the IEEE-EMBS Early Career Achievement Award, and in 1995 he received a Certificate of Appreciation from RESNA for his contributions to rehabilitation engineering and assistive technology. $\mathrm{He}$ is a member of the Board of Directors of RESNA, Chair of the RESNA Technical Guidelines Committee, and a member of the RESNA/ANSI and ISO Wheelchair Standards Committees. He is a Region 2 Representative of the IEEE-EMBS AdCom. He is a Trustee of the Paralyzed Veterans of America Spinal Cord Research Foundation and a former member of the Board of Directors for the Keystone Chapter. He was a Consultant to the Office of the Vice President of the United States under Danforth Quayle. In 1988, he was a bronze medalist in the Paralympic Games, Seoul, Republic of Korea. He was on the Steering Committee for the 1996 Paralympic Scientific Congress held in Atlanta, GA.

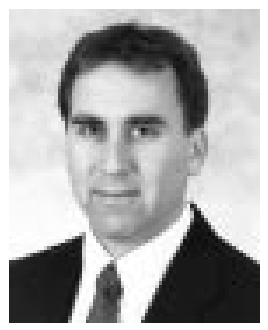

Michael L. Boninger received both the medical doctorate and the degree in mechanical engineering from the Ohio State University, Columbus. He received his specialty training in physical medicine and rehabilitation at the University of Michigan Medical Center, Ann Arbor.

He is an Assistant Professor in the Department of Orthopaedic Surgery, Division of Physical Medicine and Rehabilitation, and in the Department of Rehabilitation Science and Technology, Pittsburgh, PA. He served as Chief Resident at the University of Michigan Medical Center. After his residency program, he completed an NIDRR Fellowship in Assistive Technology at the University of Pittsburgh. He serves as Medical Director for the Center for Assistive Technology at the University of Pittsburgh Medical Center, Pittsburgh, PA. This clinic incorporates many disciplines in order to provide patients with the most appropriate assistive technology (rehabilitation engineering, occupational therapy, physical therapy, and rehabilitation medicine). He serves as the Medical Director of the Human Engineering Research Laboratories, Pittsburgh, PA. His research interests include causes of upper extremity pain in individuals who rely on manual wheelchairs for mobility, fall prevention in the elderly, wheelchair biomechanics, and appropriate utilization of assistive technology.

Dr. Boninger has received a National Institutes of Health, Clinical Investigative Development Award through the National Center for Medical Rehabilitation Research. 


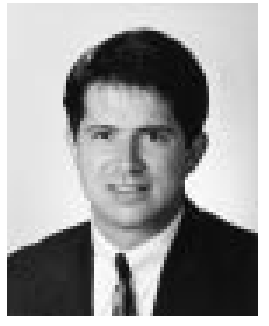

David P. VanSickle received the M.S. degree in biomedical engineering from California State University, Sacramento (CSUS), in 1994 and the B.S. degree in mechanical engineering from the University of Kansas, Lawrence, in 1991. He is currently pursuing the Ph.D. degree in bioengineering at the University of Pittsburgh, Pittsburgh, PA. In the fall of 1997, he will enter the University of Pittsburgh School of Medicine, Pittsburgh, PA.

$\mathrm{He}$ is currently a Research Associate with the School of Health and Rehabilitation Sciences following a predoctoral fellowship. His current research interests include wholebody vibration experienced by individuals who use wheelchairs and upperbody kinetics applied to manual wheelchair propulsion. He has been the author of more than 20 refereed publications including journal articles, and conference articles while working with the Human Engineering Research Laboratorits at both Pitt and CSUS.

Mr. VanSickle is a member of several professional societies, including RESNA and IEEE-EMB. He has been a finalist once and twice won RESNA/Whitaker student paper competitions. He has also been a finalist in an IEEE-EMB student paper competition.

Rick N. Robertson (M'93) received the B.Phys.Ed. degree from Ottawa University, Ottawa, Ont., Canada, in 1975, and the Master of Human Kinetics degree from Windsor University, Windsor, Ont., Canada, in 1979. He received the Ph.D. degree in neuromuscular biomechanics in the Department of Kinesiology from the University of Illinois, Urbana-Champaign, in 1985.

From 1983 to 1990, he taught at the University of Oregon, Eugene, and directed both the Human Anatomy and the Muscle Mechanics Laboratories. From 1990 to 1994, he taught in the Departments of Health and Physical Education and Biomedical Engineering at California State University, Sacramento. From 1995 to 1996, he was the Research Director for the Division of Physical Medicine and Rehabilitation, Department of Orthopedic Surgery, University of Pittsburgh Medical Center, Pittsburgh, PA. Currently, he is Director of the Biomechanics and Human Engineering Research Laboratories, California State University, Sacramento. He is also Biomechanics Director at the Human Engineering Research Laboratories, Highland Drive VA Medical Center, Pittsburgh, PA. His research interests are in the areas of wheelchair propulsion biomechanics, ergonomic factors in the development of carpal tunnel syndrome, and upper extremity injury mechanisms.

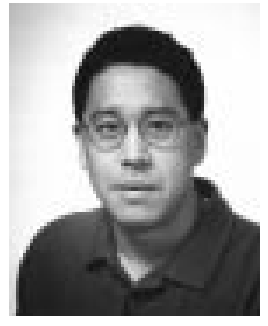

Sean D. Shimada received the B.S. degree in exercise science from the University of California, Davis, in 1992 and the M.S. degree in physical education/exercise physiology from the California State University, Sacramento (C.S.U., Sacramento), in 1994. Both of the degrees were earned with a concentration on biomechanics. He is currently pursuing the $\mathrm{Ph} . \mathrm{D}$. degree in rehabilitation science and technology and a second M.S. degree in bioengineering from the University of Pittsburgh, Pittsburgh, PA

His research focus at C.S.U., Sacramento, was on three-dimensional kinematic and kinetic analysis of wheelchair propulsion. His dissertation research is focused on developing an improved model used to describe wrist biomechanics during wheelchair propulsion. This model was developed in order to investigate the mechanisms that may cause carpal tunnel syndrome in manual wheelchair users. 